\title{
PERFIL SOCIODEMOGRÁFICO DA ENFERMAGEM INTENSIVISTA E SUAS RELAÇÕES COM ENGAJAMENTO E WORKAHOLISM
}

\section{ARTIGO ORIGINAL}

LIMA, Douglas Bertoloto ${ }^{1}$

FREITAS, Clarissa Pinto Pizarro de ${ }^{2}$

LIMA, Douglas Bertoloto. FREITAS, Clarissa Pinto Pizarro de. Perfil sociodemográfico da enfermagem intensivista e suas relações com engajamento e workaholism. Revista Científica Multidisciplinar Núcleo do Conhecimento. Ano 05, Ed. 12, Vol. 05, pp. 206-220. Dezembro de 2020. ISSN: 24480959, Link de acesso: https://www.nucleodoconhecimento.com.br/saude/engajamento-eworkaholism

\section{RESUMO}

O objetivo deste estudo foi analisar como as variáveis sociodemográficas explicam os níveis de engajamento e workaholism no trabalho de profissionais de enfermagem intensivistas. Adotou-se como método um estudo exploratório, com abordagem quantitativa dos dados, realizado com uma amostra não-probabilística de profissionais de enfermagem atuantes em Serviços de terapia intensiva adulto de hospitais públicos e privados do Estado do Rio de Janeiro. Foram realizadas análises descritivas dos participantes e correlações de Pearson entre as variáveis exploradas por meio do software Statistical Package for Social Sciences versão 19.0. Os resultados demostraram que as variáveis sociodemográficas se relacionaram de forma fraca ou

\footnotetext{
${ }_{1}^{1}$ Mestre em Psicologia Social pela Universidade Salgado de Oliveira; Mestre em Terapia Intensiva pelo Ibrati; Especialista em Enfermagem Cárdio - Intensiva; Especialista em enfermagem oncológica; graduado em Enfermagem.

2 Orientadora. Doutora em Psicologia.
} 
até mesmo não se relacionaram com o engajamento e o workaholism. Conclui-se que a carga horária semanal de trabalho se relacionou positivamente com os níveis de remuneração dos participantes, e essa com a escolaridade dos mesmos. Observouse ainda, que a os níveis de escolaridade dos profissionais de enfermagem intensivistas não estabeleceu relação estatística significativa com os índices engajamento dos participantes.

Palavras-Chave: Enfermagem, terapia intensiva, engajamento, workaholism, Saúde do Trabalhador.

\section{INTRODUÇÃO}

Na sociedade atual as pessoas investem um percentual amplo de seu tempo dedicado ao trabalho, ou até mesmo, preparando-se para esse fim. O trabalho ocupa lugar central na vida dos indivíduos sendo considerado uma atividade saudável, capaz de proporcionar as pessoas sensações de bem-estar, felicidade e satisfação, porém a relação do trabalhador com seu labor também pode resultar em desfechos negativos (DUARTE, 2018). Os desfechos que irão suceder-se frente ao trabalhador podem ser resultado de dois estados distintos de bem-estar afetivo.

Há muitas denominações acerca do construto bem-estar desde o início dos estudos que o cercam, a partir da década de 1960. Felicidade, satisfação e afetos positivos estão entre as designações mais comuns encontradas na literatura. O bem-estar está ligado a forma como as pessoas pensam e como se sentem em relação a suas vidas, sendo estruturado pelos componentes afetivo e cognitivo. O componente afetivo está ligado as emoções, como o prazer e o desprazer, e o cognitivo permite ao indivíduo uma análise mais holística de sua vida (RYAN et al., 2001). Abordaremos aqui duas formas distintas bem-estar afetivo no trabalho, o engajamento e o workaholism.

O engajamento é definido como um estado psicológico positivo em relação ao trabalho, caracterizado por vigor, dedicação e absorção. Engajamento no trabalho é considerado uma forma prazerosa que as pessoas vivenciam ao lidar com seu trabalho, culminando em melhores desempenho e resultados organizacionais. 
Profissionais engajados mostram-se motivados e com iniciativa para o trabalho e buscam adaptar-se as dificuldades do ambiente de trabalho, algo essencial aos profissionais de enfermagem atuantes nos ambientes de terapia intensiva (SCHAUFELI, 2017).

No entanto, essa dedicação pode chegar as vias do exagero, levando o indivíduo a uma vida centrada no trabalho e um maior envolvimento com a organização, gerando prejuízos de cunho pessoal. O termo workaholism é utilizado para descrever este envolvimento excessivo com o trabalho. É descrito na literatura a associação positiva entre burnout e workaholism (ZEIJEN et al., 2018).

A síndrome de Burnout é uma resposta ao estresse ocupacional crônico, que se caracteriza pela exaustão emocional, despersonalização e reduzida realização profissional (SCHAUFELI, 2017). No contexto de trabalho da enfermagem, os profissionais diretamente envolvidos na assistência aos pacientes são os mais acometidos por essa síndrome. Burnout e engajamento são considerados conceitos opostos, devendo inclusive serem medidos de forma independente (ROTTA et al., 2019). Baseado nessas afirmativas, configura-se a hipótese um deste estudo: a variável sociodemográfica "escolaridade" estará positivamente associada aos índices de engajamento.

Entre os profissionais da saúde, os trabalhadores da enfermagem são os que permanecem a maior parte do tempo em ao lado dos pacientes, estando presente e vivenciando as mais diversas e complexas situações. Carga horária semanal excessiva, escassez de recursos humanos e materiais, relações de conflito e ambiguidade de papéis, redução na percepção e apoio social são listados como os estressores mais comuns encontrados no ambiente de trabalho destes profissionais (FANG, 2017).

As condições de trabalho dos profissionais de enfermagem podem ser examinadas nos moldes do Modelo de Demanda e Recursos (Job Demands and Resources Model - JDR). O modelo de demandas e recursos do trabalho propõe que os ambientes de trabalho podem iniciar dois processos distintos, o de comprometimento da saúde e o 
motivacional. O processo motivacional tem início com recursos do trabalho adequados, que incentivam os funcionários a atingir suas metas relacionadas ao trabalho. Em contrapartida, o processo de comprometimento da saúde inicia-se com demandas de trabalho consistentemente elevadas que podem esgotar os recursos energéticos dos colaboradores, levando-os à fadiga e a problemas de saúde (BAKKER et al., 2017).

A unidade de terapia intensiva compõe-se de um ambiente destinado a permanência de pacientes em estado crítico, que necessitam de profissionais com competências específicas. A atuação dos profissionais de enfermagem nesse ambiente pode ser de elevado custo emocional, físico e psíquico, resultando em indivíduos exaustos, menos tolerantes e irritadiços (SOUZA et al., 2019).

Estudo desenvolvido em contexto nacional demonstrou que a força de trabalho na enfermagem é relativamente jovem e continua rejuvenescendo. Dados afirmam que $40 \%$ do contingente pesquisado tem entre $36-50$ anos e outros $38 \%$ estão na faixa etária situada entre os 26-35 anos (MACHADO et al., 2016).

A idade é considerada por alguns autores como um fator preditivo para a ocorrência de workaholism, associando a jovialidade como um atributo a fomentar a incapacidade de parar de trabalhar. Sob essa ótica, sugere-se que o workaholism diminua à medida que o indivíduo se torne mais experiente, evolua na carreira ou até mesmo estabeleça relacionamentos (ZEIJEN et al., 2018). Essas afirmativas estimularam a criação da segunda hipótese proposta: a variável sociodemográfica "idade" estará positivamente associada aos níveis de workaholism.

A jornada de trabalho é algo inerente a vida de qualquer trabalhador, entretanto, em alguns países estudos demonstram que o trabalhador da área da enfermagem possui longas jornadas de trabalho, que associados a fatores como a baixa remuneração elevam a percepção das demandas físicas e emocionais (OLIVEIRA et al., 2018). Ao longo dos anos, muitas definições surgiram buscando conceituar o workaholism, boa parte delas buscaram associar exclusivamente esse construto ao número excessivo de horas trabalhadas semanalmente. 
Mais recentemente, compreendeu-se o workaholism como um fenômeno psicossocial multifacetado, constituído pelas dimensões de trabalho compulsivo e trabalho excessivo, onde o profissional têm dificuldade de parar de pensar no seu trabalho ou mesmo de se afastar dele fisicamente (SCHAUFELI, 2017).

Profissionais workaholics não conseguem resistir ao impulso compulsivo em trabalhar, diferindo-se dos trabalhadores engajados, que percebem o labor como algo desafiador e alegre (ROTTA et al., 2019). Esse cenário originou a terceira hipótese explorada: a variável sociodemográfica "carga horária semanal" não estabelecerá associações estatísticas significativas com os níveis de workaholism e engajamento.

Ainda que reconhecida sua importância, nota-se que escassez de estudos nesta temática envolvendo profissionais de enfermagem intensivistas, especialmente no cenário nacional. E ante esta realidade nos perguntamos: existe relação entre as características sociodemográficas e os níveis bem-estar no trabalho de profissionais de enfermagem intensivistas?

Frente a essas questões, o objetivo deste estudo foi analisar como as variáveis sociodemográficas explicam os níveis de engajamento e workaholism no trabalho de profissionais de enfermagem intensivistas.

\section{MÉTODO}

Trata-se de um estudo exploratório, com abordagem quantitativa, realizado com uma amostra não-probabilística de profissionais de enfermagem atuantes em Serviços de Terapia Intensiva Adulto de hospitais públicos e privados, localizados na cidade do Rio de Janeiro e região metropolitana do Estado do Rio de Janeiro. Os sujeitos da pesquisa foram 122 profissionais de enfermagem - enfermeiros, técnicos e auxiliares de enfermagem.

O grupo de profissionais foi acessado pelo método de coleta de dados presencial e on-line. A todos os abordados presencialmente, foi oferecido o Termo de Consentimento Livre e Esclarecido (TCLE). Após assinado, os participantes tiveram 
acesso ao questionário sociodemográfico e laboral, e as duas escalas. A coleta on line foi realizada via site "Survey Monkey", e seguiu as mesmas exigências.

Foram realizados os procedimentos éticos conforme Resolução 196 do Conselho Nacional de Saúde (Ministério da Saúde, 1996), no que diz respeito à pesquisa com seres humanos. O Presente estudo foi avaliado sob o parecer consubstanciado da Universidade Salgado de Oliveira sob número 2.998.481 e CAEE: 00379718.9.1001.5289.

Na coleta de dados foram aplicadas:

a) Questionário Sociodemográfico e Laboral. Este instrumento investigou informações Sociodemográficas e laborais dos profissionais, tais como gênero, idade, estado civil, escolaridade, vínculo trabalhista, carga horária semanal, remuneração entre outros dados;

b) Na avaliação dos níveis de workaholism, foi utilizada a Dutch Workaholism Scale (DUWAS-10); A escala avalia a adição ao trabalho em suas duas principais dimensões, o Trabalho Compulsivo (TC) e o Trabalho Excessivo (TE). No total, constitui-se de 10 itens avaliados por uma escala tipo likert; A Dutch Workaholism Scale (DUWAS-10), no contexto brasileiro, foi adaptada por VASQUEZ et al., (2018). As análises da versão reduzida DUWAS-10 sugerem que tanto a estrutura unidimensional (Adição) (c2(gl) $=207,60$ (35); $\mathrm{p}<0,001$; $\mathrm{CFI}=0,93$; $\mathrm{TLI}=0,91$; RMSEA $(90 \%$ l.C. $)=0,09(0,08-0,10))$ ou de dois fatores oblíquos (Trabalhar Excessivamente e Trabalhar Compulsivamente) são adequadas (2(gl) = 169,68 (34); $\mathrm{p}<0,001 ; \mathrm{CFI}=0,94 ; \mathrm{TLI}=0,93 ; \mathrm{RMSEA}(90 \% \mathrm{l} . \mathrm{C}$. $)=0,08(0,07-0,10)$

c) Os níveis de engajamento foram avaliados pela Escala de Engajamento no Trabalho Utrecht (Utrecht Work Engagement Scale) (UWES). A UWES possui 17 itens distribuídos nas três dimensões do engajamento: vigor (6 itens), dedicação (5 itens) e absorção ( 6 itens). O questionário é respondido em uma escala Likert de sete pontos $(0=$ Nunca/, nenhuma vez a $6=$ Sempre, todos os dias). Os índices de consistência 
interna da versão original do questionário apresentaram-se adequados (Vigor, $\alpha=$ 0,80; Dedicação $\alpha=0,91$ e Absorção $\alpha=0,7$ ), (SCHAUFELI et al., 2002).

No procedimento de análise dos dados foi realizado com auxílio do programa Statistical Package for Social Sciences - SPSS, versão 19.0, procedendo-se com a análise descritiva de caráter exploratório, no intuito de avaliar a distribuição dos itens, casos omissos e identificação de extremos. Analisou-se a possível existência de subgrupos ou de algumas especificidades entre os participantes e análise de frequência através do Histograma de Frequências para identificar as características e a distribuição dos dados. Foram calculados, ainda, o desvio padrão e as médias, para identificação do comportamento geral da amostra.

$\mathrm{Na}$ busca da compreensão das relações entre as dimensões das variáveis e entre as próprias variáveis, foi realizada a Correlação de Pearson. O cruzamento dessas relações ocorreu entre as dimensões vigor, dedicação e absorção do engajamento, as dimensões trabalho excessivo e trabalho compulsivo do workaholism.

\section{RESULTADOS}

Um maior percentual de indivíduos $(38,5 \% ; n=47)$, tem entre 27 e 37 anos. Foi observado que $36,0 \%(n=44)$ são enfermeiros, $57,3 \%(n=70)$ técnicos de enfermagem e $6,6 \%(n=8)$ auxiliares de enfermagem. O total de $60 \%(n=73)$ trabalhadores afirmaram ter até 10 anos de profissão, 56,6\% $(n=69)$ responderam não ter outro vínculo trabalhista. Houve prevalência dos profissionais que afirmaram trabalhar entre 40 e 50 horas semanais. Um maior percentual de indivíduos apresentou escolaridade de nível médio. Alguns resultados das características sociodemográficas são apresentados na tabela 1.

Tabela 1 - Características dos profissionais de enfermagem participantes do estudo. Rio de Janeiro, 2019.

Variáveis

N $\%$




\begin{tabular}{|c|c|c|}
\hline \multicolumn{3}{|l|}{ Sexo } \\
\hline Feminino & 84 & 68,9 \\
\hline Masculino & 38 & 31,1 \\
\hline \multicolumn{3}{|l|}{ Estado civil } \\
\hline Solteiro & 45 & 36,9 \\
\hline Casado & 47 & 38,5 \\
\hline Divorciado/viúvo & 30 & 24,6 \\
\hline \multicolumn{3}{|l|}{ Escolaridade } \\
\hline Nível técnico & 32 & 45,9 \\
\hline Graduado & 42 & 34,4 \\
\hline Especialista & 23 & 18,9 \\
\hline Mestre & 01 & 0,8 \\
\hline \multicolumn{3}{|l|}{ Categoria } \\
\hline Enfermeiro & 44 & 36,0 \\
\hline Técnico de enfermagem & 70 & 57,3 \\
\hline Auxiliar de enfermagem & 08 & 6,6 \\
\hline Continua Estudando & & \\
\hline Sim & 32 & 26,2 \\
\hline Não & 69 & 56,6 \\
\hline
\end{tabular}




\begin{tabular}{|l|l|l|}
\hline $\begin{array}{l}\text { Não responderam } \\
\text { Carga horária semanal }\end{array}$ & 21 & 17,2 \\
\hline Entre $\mathbf{3 0}$ e $\mathbf{3 5}$ horas & 37 & 30,3 \\
\hline Entre 35e $\mathbf{4 0}$ horas & 21 & 17,2 \\
\hline $\begin{array}{l}\text { Mais de } \mathbf{6 0} \text { horas } \\
\text { Remuneração }\end{array}$ & 47 & 38,5 \\
\hline 1-2 salários-mínimos & 10 & 9,0 \\
\hline 2-3 salários-mínimos & 24 & 19,7 \\
\hline 3-4 salários-mínimos & 14 & 11,5 \\
\hline
\end{tabular}

Fonte: Elaboração dos autores.

A variável sociodemográfica "escolaridade" apresentou correlação de magnitude fraca e negativa com os níveis de engajamento dos participantes $(r=-0,21 ; p>0,05)$. Os índices de workaholism, embora médios $(M=1,9 ; D P=0,5)$, não apresentaram correlação estatisticamente significativa com a variável sociodemográfica "Idade", conforme detalhado na tabela 2. A variável sociodemográfica "carga horária semanal" não estabeleceu relação estatisticamente significativa com as variáveis de bem-estar afetivo workaholism e engajamento.

Tabela 2. Correlações entre as variáveis sociodemográficas e do trabalho.

\begin{tabular}{|l|l|l|l|l|l|l|l|l|}
\hline & $M$ & DP & 1 & 2 & 3 & 4 & 5 & 6 \\
\hline 1 -Idade & 34,2 & 8,3 & & & & & & \\
\hline 2-C.H.S. & 44,5 & 1,2 & $0,21^{* \star}$ & & & & & \\
\hline 3-Escolaridade & 2,9 & 0,9 & $0,29^{* \star}$ & 0,05 & & & & \\
\hline 4-Remuneração & 2,4 & 1,8 & $0,21^{* \star}$ & $0,31^{* \star}$ & $0,66^{\star *}$ & & & \\
\hline
\end{tabular}




\begin{tabular}{|l|l|l|l|l|l|l|l|l|}
\hline 5-Engajamento & 3,3 & 2,9 & 0,04 & 0,04 & $-0,21^{\star *}$ & $0,36^{\star \star}$ & & \\
\hline 6-Workaholism & 1,9 & 0,5 & $-0,01$ & 0,01 & 0,06 & 0,05 & $-0,01$ & 0,03 \\
\hline
\end{tabular}

Note: ${ }^{* *} p<0,001 ;{ }^{*} p>0,05$. C.H.S $=$ carga horária semanal.

Fonte: Elaboração dos autores.

\section{DISCUSSÃO}

Dos 122 participantes deste estudo, 36,0\% ( $n=44)$ exercem a profissão de enfermeiro, porém, $65(52,0 \%)$ participantes declararam possuir escolaridade ao nível de graduação, especialista ou mestre. Esse fato demonstra que uma grande fração de profissionais técnicos de enfermagem possuem qualificação acima das necessidades exigidas para função que exercem.

O Engajamento é considerado como um estado de espírito positivo, satisfatório e relacionado ao trabalho, que envolve comprometimento e alinhamento do profissional com o ambiente e a atividade laboral. Trata-se de um estado de bem-estar afetivo, persistente e abrangente, de natureza motivacional e social, caracterizado por três dimensões: Vigor, Absorção e Dedicação (SCHAUFELI, 2017).

Neste estudo, observou-se que as médias deste construto se mostraram elevadas $(M=3,3 ; D P=2,90)$, consideradas todo o conjunto de profissionais participantes. Tendo em vista esses valores e o fato de associação fraca e negativa entre as variáveis exploradas, podemos inferir que o engajamento nos profissionais de enfermagem intensivistas não é fortemente determinado por sua qualificação, o que não permite corroborar a hipótese um deste estudo. Em um segmento profissional onde os profissionais de nível médio respondem pela maior parte do contingente de trabalhadores, esse resultado evidencia um ganho potencial, dado todo comprometimento e envolvimento com o trabalho dos participantes.

Simultaneamente, registrou-se uma associação forte e positiva entre os índices de escolaridade e remuneração $(r=0,66 ; p>0,05)$. Profissionais de enfermagem 
percebem que a medida que buscam maior qualificação, surgem novos desafios e oportunidades, o que resulta em maiores ganhos salariais.

Dentro do hospital as unidades assistenciais possuem características específicas, que variam de acordo com o tipo de atendimento realizado, o perfil dos pacientes e o tempo de permanência destes. O contexto da terapia intensiva exige do profissional de enfermagem alta performance, o que pode resultar em maior envolvimento com o trabalho, independentemente de seu cargo (OLIVEIRA et al., 2018).

Trabalhadores engajados trabalham e agem de maneira proativa, são concentrados nos objetivos que pretendem atingir e estão em consonância com o que é bom para a organização e para seus clientes. Normalmente são persistentes, ainda que o trabalho não esteja seguindo de acordo com o planejado (BAKKER et al., 2017).

Um maior percentual de participantes relatou ter entre 27 e 37 anos ( $n=47 ; 38,5 \%)$, proporção aproximada a estudo nacional que verificou o perfil sociodemográfico da enfermagem brasileira (MACHADO et al., 2016). Nesta fase da vida laboral, os indivíduos estão em plena atividade de suas funções cognitivas, técnicas e práticas, exercendo papel importante no mercado de trabalho e estando dispostos a trabalhar por longas horas e períodos. Entretanto, nossos resultados não permitem corroborar a segunda hipótese proposta, que previa que o workaholism seria mais prevalente nessa faixa etária.

Estudo nacional recente avaliou a idade dos profissionais de enfermagem e sua associação com os índices de engajamento no trabalho e revelou que profissionais situados na faixa etária até 34 e acima dos 40 anos demonstraram estar mais engajados. (GARBIN et al., 2019). Em análise paralela, observamos que 62,5\% ( $n=$ 30) dos profissionais com idade até 37 anos afirmaram continuar estudando, buscando maiores níveis de escolaridade.

Tal panorama associado aos altos índices de engajamento encontrados neste estudo, ajuda a compreender esse grupo de intensivistas como engajado e não workaholics, pois justificam seu maior empenho ao labor em busca de melhores salários. $O$ 
workaholism caracteriza-se pela compulsão ou a necessidade incontrolável de trabalhar incessantemente, entretanto, essa necessidade não é saciada ou direcionada aos ganhos materiais. O workaholism é um fenômeno Psicossocial relativamente recente, porém com consequências muito negativas a saúde do trabalhador (ZEIJEN et al., 2018).

A variável sociodemográfica "carga horária semanal" não estabeleceu relação estatisticamente significativa com as variáveis de desfecho workaholism e engajamento, corroborando a hipótese 3 deste estudo. Conceitualmente, carga horária semanal é o número de horas resultantes da soma das jornadas nos dias úteis da semana (BRASIL, 1988). Existe uma regra estabelecida de 08 horas diárias, no limite de 44 horas semanais, devendo ser observadas eventuais reduções por força de contrato.

Cabe ressaltar que o Conselho Federal de Enfermagem (Cofen), por meio da resolução n. 293/2004, regulamentou jornada de 36 horas semanais para atividade assistencial e de 40 horas semanais para atividades administrativas. Nos últimos 20 anos essa categoria luta por uma jornada máxima de trinta horas semanais, em torno do projeto de lei n. 2.295/2000 (OLIVEIRA et al., 2018).

Efetivamente, o que fizemos no questionamento sociodemográfico e laboral deste estudo, foi investigar a carga horária semanal de trabalho dos profissionais de enfermagem atuantes nos serviços de terapia intensiva. Descobriu-se que, em média, $38,5 \%(n=47)$ afirmaram trabalhar entre 40 e $50 \mathrm{~h}$ semanais e $9 \%(n=10)$ acima de 60 horas semanais. Destacamos que essa jornada de trabalho total declarada, não faz menção ou inclusão a horas extras trabalhadas dentro das próprias instituições hospitalares, referem-se somente aos contratos de trabalho oficiais vigentes.

Ao longo dos últimos 40 anos surgiram algumas definições acerca do workaholism, que restringiram o fenômeno a aqueles trabalhavam mais de 50 horas por semana. Considerando que o trabalho contemporâneo é marcado pela competitividade e pela participação do trabalhador, é evidente que grande parte da força de trabalho da enfermagem poderia encaixar-se perfeitamente nessa definição. 
Entretanto, considerar exclusivamente o número de horas trabalhadas é inadequado, uma vez que a relação do indivíduo com o trabalho é mais representativa do que apenas isso, e o workaholism vem demonstrando ser um fenômeno multifatorial complexo (ZEIJEN et al., 2018). As demandas de trabalho para os profissionais de enfermagem no contexto da terapia intensiva são múltiplas e estão envoltas a tomar muitas decisões rápidas, observar continuamente os pacientes, e muitas vezes cuidar de familiares com necessidades emocionais (SANTOS et al., 2007).

Os Workaholics investem muitos esforços no trabalho, sejam eles físicos ou psicológicos, o que os deixa com menos recursos para dedicar as suas famílias, resultando em sacrifícios na vida pessoal. Na prática profissional, o workaholism é associado como um preditor potencial da Síndrome de Burnout (ZEIJEN et al., 2018).

Normalmente esse processo decorre de um esforço adicional no trabalho, onde o colaborador emprega quantidades excessivas de energia, podendo essa resposta variar de indivíduo para indivíduo. Em ambientes de trabalho críticos como o de terapia intensiva, o enfermeiro busca executar funções procurando fazer o melhor que pode, às vezes até a exaustão, para atingir as metas necessárias e prestar uma assistência de qualidade (CRUZ et al., 2008).

É oportuno mencionar que as variáveis sociodemográficas "carga horária semanal" e "remuneração" estabeleceram relação estatisticamente positiva e significativa ( $r=$ $0,31 ; p>0,05)$. Essa condição evidencia que profissionais de enfermagem buscam melhores salários às custas de um aumento significativo e degradante da carga horária semanal de trabalho, como informam outros estudos realizados em contexto nacional (MACHADO et al.; 2016; OLIVEIRA et al., 2018). Esses resultados vêm reafirmar a desvalorização dos profissionais de enfermagem, mesmo em um cenário tão rodeado de especificidades e necessidade de especialização quanto o de terapia intensiva (OLIVEIRA et al., 2018; SILVA et al., 2013).

Os profissionais de enfermagem reconhecem o ambiente hospitalar como um local onde é necessário manter sob vigilância e cuidados vidas frágeis, e que para tal, conhecimento, habilidade e competência técnicas associadas ao controle emocional 
são essenciais para manutenção dessas vidas. Nas unidades críticas, como os serviços de terapia intensiva, alguns fatores são comumente relatados como desfavoráveis nos processos de trabalho, como alarmes emitidos pelos monitores cardíacos, bombas de infusão e ventiladores mecânicos, conversas em alto tom nos corredores, abertura e fechamento de portas de forma violenta e quedas de objetos, além do excesso de tráfego de pessoas na unidade (SANTOS et al., 2007). Esses fatores podem elevar a demanda de trabalho dos profissionais de enfermagem atuantes nos serviços de terapia intensiva.

Do ponto de vista conceitual, há de ressaltar-se que existe uma importante diferença entre demanda e carga horária semanal. As demandas de trabalho retratam os aspectos físicos, psicológicos, organizacionais e sociais do ambiente de trabalho e denotam empenho físico e ou esforço psicológicos e cognitivos, portanto, estão associados a custos para os colaboradores (BAKKER et al., 2017).

As próprias características do trabalho da enfermagem requerem múltiplas demandas, que são resultantes da complexidade dos cuidados prestados, do próprio ambiente laboral e das exigências oriundas tanto da prestação de cuidados aos pacientes quanto do próprio hospital. Desta forma, estabelecemos uma limitação deste estudo, visto que não foram exploradas as características das demandas de trabalho dos profissionais de enfermagem intensivistas, não permitindo realizar associações delas com as variáveis analisadas.

\section{CONCLUSÃO}

O objetivo deste estudo foi analisar como as variáveis sociodemográficas explicam os níveis de engajamento e workaholism no trabalho de profissionais de enfermagem intensivistas. Observou-se que a escolaridade, a idade e a carga horária semanal dos participantes, não estabeleceram relações estatisticamente significativas com as variáveis de desfecho exploradas.

Entretanto, algumas associações em paralelo foram estatisticamente significativas, e precisam ser valorizadas no intuito de preservar a saúde dos profissionais de 
enfermagem atuantes nos serviços de terapia intensiva. A escolaridade não se relacionou positivamente com os níveis de engajamento, mas apresentou associação positiva com a carga horária semanal, e esta por sua vez com os níveis de remuneração. Isso nos levou a entender que os profissionais trabalham por mais tempo em troca de um salário digno, reduzindo seu tempo de lazer e convívio familiar e social.

Esse contexto evidencia a necessidade de valorização do profissional de enfermagem intensivista no mercado de trabalho. Entende-se, portanto, que a definição legal de uma jornada de trinta horas de trabalho semanal poderá fortalecer a enfermagem ciência, e ainda preservar a saúde destes profissionais.

Embora tenha apresentado níveis médios, o workaholism não se associou a idade dos participantes, fator positivo dado as características da amostra em questão. Entretanto, percebeu-se a necessidade de explorar e diferenciar conceitualmente demandas de trabalho de carga horária semanal. Fato que sugere novas pesquisas nesse campo. Não foram percebidas quaisquer associações entre engajamento e workaholism, o que fortalece a ideia de construtos distintos.

O avanço do conhecimento científico neste campo pode ser um pilar com intuito de futuramente chegar a oferecer ascendência sobre as políticas públicas que visem auxiliar para melhoria da saúde do trabalhador. Consideramos plenamente atingidos os objetivos deste estudo, e que o mesmo vem a contribuir na literatura científica para melhor compreensão do fenômeno emergente workaholism e somar a outros existentes quanto ao construto engajamento.

\section{REFERÊNCIAS}

AZEVEDO, Bruno Del Sarto; NERY, Adriana Alves; CARDOSO, Jefferson Paixão. OCCUPATIONAL STRESS AND DISSATISFACTION WITH QUALITY OF WORK LIFE IN NURSING. Texto contexto - enferm., Florianópolis, v. 26,n. 1, e3940015, 2017

Available from 
<http://www.scielo.br/scielo.php?script=sci_arttext\&pid=S0104-

07072017000100309\&Ing=en\&nrm=iso>. access on 08 Oct. 2020. puMar27, 2017.

BAKKER, Arnold; DEMEROUTI, Evangelia. Job Demands-Resources Theory: Taking Stock and Looking Forward. Journal of Occupational Health Psychology, 2017. Vol. 22, (3), 273-285

BANDURA, Albert. Self-efficacy: the exercise of control. 1997, New York: W.H. Freeman

BRASIL. Constituição federal (1988). Constituição da República Federativa do Brasil. Brasília, DF: Senado, 1988

CAMELO, Henriques; SILVA, Vânea Lúcia dos Santos; LAUS, Ana Maria; \& CHAVES, Luciele Dias Pedreschi. Perfil profissional de enfermeiros atuantes em unidades de terapia intensiva de um hospital de ensino. Ciencia y enfermeria, 2013.19(3), $51-62$

CARLOTTO, Mary Sandra. Adição ao trabalho e relação com fatores de risco sociodemográfi cos, laborais e psicossociais. Psico-USF, 2011. 16(1), 87-95.

CRUZ, Elisa; SOUZA, Norma Valéria Dantas Oliveira. Repercussão da variabilidade na saúde do enfermeiro intensivista. Revista eletrônica de enfermagem, 2008 . 18(1):1102-13.

DUARTE, Maria de Lucia Custódio; GLANZENR Cecília Helena; PEREIRA LETÍCIA PASSOS. O trabalho em emergência hospitalar: sofrimento e estratégias defensivas dos enfermeiros. Revista Gaúcha de Enfermagem, 2018; 39: e20170255.

FANG, Ya Xuan. Burnout and work-family conflict among nurses during the preparation for reevaluation of a grade a tertiary hospital. Chinese Nursing Research, 2017.4(1), 51-55. 
GARBIN, Keiti et al. A Idade como Diferencial no Engagement dos Profissionais de Enfermagem. Psic.: Teor. e Pesq., Brasília , v. 35 e35516, 2019. Available from $37722019000100615 \& \operatorname{lng}=e n \& n r m=i s o>$.

LACCORT, Alessandra de Almeida; DE OLIVEIRA, Grasilela Backer. A importância do trabalho em equipe no contexto da enfermagem. Revista Uningá REVIEW, [S.I.], v. 29, n. 3, mar. 2017. ISSN 2178-2571.

MACHADO, Maria Helena et al. Mercado de trabalho em enfermagem no âmbito do SUS: uma abordagem a partir da pesquisa Perfil da Enfermagem no Brasil. Divulgação Saúde Debate, 2016.56: 52-69.

OLIVEIRA, Bruno Luciano Carneiro Alves de; SILVA, Alécia Maria da; LIMA, Sara Fiterman. Carga Semanal de Trabalho Para Enfermeiros no Brasil: Desafios para o exercício da profissão. Trab. educ. saúde, Rio de Janeiro, v. 16, n. 3,p. 1221-1236, Dec. 2018

OLIVEIRA, Lucia Barbosa de; ROCHA, Juliana da Costa. Engajamento no trabalho: antecedentes individuais e situacionais e sua relação com a intenção de rotatividade. Rev. bras. gest. Neg., São Paulo, v. 19, n. 65p. 415-431, Sept. 2017 .

ROTTA, Daniela Salgagni et al., Engagement of multi-professional residents in health. Rev Esc Enferm USP. 2019;53:e03437.

SANTOS, José Luiz Guedes et al., Ambiente de Trabalho do Enfermeiro na Divisão de Enfermagem Materno-Infantil de um Hospital Universitário. Revista de Enfermagem do Centro-Oeste Mineiro. 2018;8: e 2099. doi: https://doi.org/10.19175/recom.v7i0.2099

SANTOS, Luciana Soares Costa; GUIRARDELLO, Edinêis de Brito. Demandas de atenção do enfermeiro no ambiente de trabalho. Revista Latino-americana de Enfermagem, 2007. janeiro-fevereiro; 15(1). 
SCHAUFELI, Wilmar. Applying the Job Demands-Resources model: A How to guide measuring and tackling work engagement and burnout. Organizational Dynamics, 2017.46 (2).120-127.

SCHAUFELI, Wilmar; SALANOVA, Maria; GONZALEZ-ROMÁ Vicent, BAKKER, Arnold. The measurement of engagement and burnout: A two sample confirmatory factor analytic approach. Journal of Happiness studies, 2002; 3(1), 71-92.

SILVA, Rômulo Botelho et al., Qualidade da assistência de enfermagem em unidade de terapia intensiva de um hospital escola. Revista Gaúcha de Enfermagem, 2013;34(4):114-120.

VAZQUEZ, Ana Claudia Souza et al. Evidências de validade da versão brasileira da escala de workaholism (DUWAS-16) e sua versão breve (DUWAS-10). Avaliação psicológica, Itatiba, v. 17,n. 1,p. 69-78, 2018 .

VIANA, Renata Andrea Pietro Pereira et al. Perfil do enfermeiro de terapia intensiva em diferentes regiões do Brasil. Texto contexto - enferm., Florianópolis , v. 23,n. 1,p. 151-159, Mar. 2014.

ZEIJEN, Marijntje; PEETERS Maria \& HAKANEN, Jari. Workaholism versus work engagement and job crafting: What is the role of self-management strategies? Human Resource Management Journal; 2018, 28:357-373. doi.org/10.1111/17488583.

Enviado: Novembro, 2020.

Aprovado: Dezembro, 2020. 